\title{
Virtually Haken surgeries on once-punctured torus bundles
}

\author{
JOSEPH D. MASTERS
}

\begin{abstract}
We describe a class $\mathcal{C}$ of punctured torus bundles such that, for each $M \in \mathcal{C}$, all but finitely many Dehn fillings on $M$ are virtually Haken. We show that $\mathcal{C}$ contains infinitely many commensurability classes, and we give evidence that $\mathcal{C}$ includes representatives of "most" commensurability classes of punctured torus bundles.

In particular, we define an integer-valued complexity function on monodromies $f$ (essentially the length of the LR-factorization of $f_{*}$ in $\left.\mathrm{PSL}_{2}(\mathbb{Z})\right)$ and use a computer to show that if the monodromy of $M$ has complexity at most 5 , then $M$ is finitely covered by an element of $\mathcal{C}$. If the monodromy has complexity at most 12 , then, with at most 36 exceptions, $M$ is finitely covered by an element of $\mathcal{C}$

We also give a method for computing "algebraic boundary slopes" in certain finite covers of punctured torus bundles.
\end{abstract}

\section{Introduction}

A compact 3-manifold $M$ is Haken if it is irreducible, and contains an orientable, essential surface. $M$ is virtually Haken if it is finitely covered by a Haken manifold. One of the central problems in 3-dimensional topology is Waldhausen's conjecture, which states that every closed, irreducible 3-manifold with infinite fundamental group is virtually Haken.

A knot manifold is an irreducible, orientable, compact 3-manifold whose boundary is a single torus. A knot manifold $M$ is small if every closed incompressible surface in $M$ is boundary parallel. A knot manifold is hyperbolic if its interior admits a complete hyperbolic metric of finite volume. A slope on a torus $T$ is a non-trivial isotopy class of simple closed curves on $T$. If $M$ is a 3-manifold, $T$ is a torus component of $\partial M$ and $\alpha$ is a slope on $T$, then $M(\alpha)$ denotes the manifold obtained from $M$ by Dehn filling along a simple closed curve representing the slope $\alpha$. We say that a knot manifold has property $\mathrm{VH}$ if $M(\alpha)$ is virtually Haken for all but finitely many 
slopes $\alpha$ on $\partial M$. An important special case of Waldhausen's conjecture is the following:

Conjecture 1.1. Let $M$ be a hyperbolic knot manifold. Then $M$ has property $\mathrm{VH}$.

Examples of small knot manifolds satisfying property $\mathrm{VH}$ have been given in $[2,5,7]$.

Let $F$ be homeomorphic to a torus with an open disk removed. We choose a basepoint, $p \in \partial F$, for $\pi_{1} F$. Let $x$ and $y$ be generators of $\pi_{1} F$, as pictured in figure 1. Let $D_{x}$ and $D_{y}$ represent Dehn twists along simple closed curves in int $F$ which are isotopic to loops representing $x$ and $y$, respectively. Let $\mathcal{M}_{1}^{1}$ denote the mapping class group of $F$; that is the group of orientation-preserving automorphisms of $F$ which restrict to the identity on $\partial F$, modulo isotopies which fix every point of $\partial F$. It is well known that $\mathcal{M}_{1}^{1}$ is generated by $D_{x}$ and $D_{y}$. There is a natural map $\phi: \mathcal{M}_{1}^{1} \rightarrow \mathrm{SL}_{2}(\mathbb{Z})$, whose kernel is generated by a Dehn twist around a curve parallel to the boundary of $F$. We sometimes use the notation $\phi(f)=f_{*}$.

Let $H_{3}=\phi^{-1}\left\langle D_{x *}, D_{y *}^{3}\right\rangle$, and let $H_{4}=\phi^{-1}\left\langle D_{x *}, D_{y *}^{4}\right\rangle$. It is a fact (see Section 7 ) that $H_{3}$ and $H_{4}$ are both finite-index subgroups of $\mathcal{M}_{1}^{1}$.

Theorem 1.2. Let $M$ be an orientable, atoroidal 3-manifold which fibers over $S^{1}$, whose fiber, $F$, is a compact, orientable surface of genus 1 , with a single boundary component. Let $f: F \rightarrow F$ be the monodromy, and suppose that the mapping class of $f$ lies in the subgroup $H_{i}$, where $i=3$ or 4 . Then there are slopes $\beta_{i}^{1}, \beta_{i}^{2}$ and an integer $N$ such that $M(\alpha)$ is virtually Haken whenever $I\left(\alpha, \beta_{i}^{1}\right)>N$ and $I\left(\alpha, \beta_{i}^{2}\right)>1$.

Since $H_{3}$ and $H_{4}$ have finite index, it follows that every hyperbolic punctured torus bundle has infinitely many virtually Haken surgeries, a

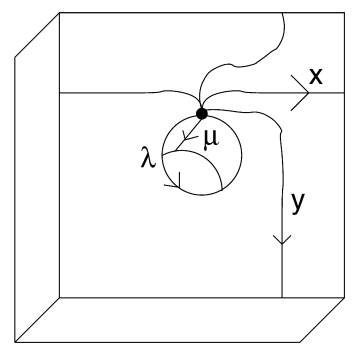

Figure 1: Notation for the bundle $M$. 
result which was first proved by Baker [1] (see also [7]). The slopes $\beta_{i}^{j}$ are computable (see Sections 6-8).

For a given monodromy $f$, let $\beta_{i}^{j}=\beta_{i}^{j}(f)$ be slopes as in Theorem 1.2. Let $\mathcal{C}$ be the set of mapping classes $f$ of $F$ such that $f \in H_{3} \cap H_{4}$ and $\left\{\beta_{3}^{1}, \beta_{3}^{2}\right\} \cap\left\{\beta_{4}^{1}, \beta_{4}^{2}\right\}=\emptyset$.

Corollary 1.3. If $f \in \mathcal{C}$, then $M$ has property $\mathrm{VH}$.

We shall show that there are infinitely many commensurability classes of once-punctured torus bundles whose monodromies lie in the class $\mathcal{C}$. Thus we obtain:

Theorem 1.4. There are infinitely many pairwise non-commensurable once-punctured torus bundles which have property $\mathrm{VH}$.

Remark. 1. In the case where $f \in H_{4}, 1.2$ is a corollary of [7, Theorem 1.3]. The proof we give here is completely different.

2. Many of the bundles in $\mathcal{C}$ have no exceptional surgeries, and so the techniques of $[2,5]$ cannot be applied.

It appears that (in some sense) "most" monodromies $f$ have a power which lies in $\mathcal{C}$, and thus most punctured torus bundles are commensurable to ones with property VH. We have verified this on a computer, for monodromies of low complexity.

To make these statements precise, we introduce a complexity function on monodromies. Recall that every element $g \in \mathrm{SL}_{2}(\mathbb{Z})$ can be written uniquely as a positive word in $D_{x *}^{-1}$ and $D_{y *}$, times $\pm \mathrm{Id}$. We define the "complexity" of $g$ to be the length of this word. Similarly, if $f \in \mathcal{M}_{1}^{1}$, then we define the complexity of $f$ to be the complexity of $f_{*}$ in $\mathrm{SL}_{2}(\mathbb{Z})$, and if $M$ is a punctured torus bundle with monodromy $f$, the complexity of $M$ is defined to be the minimum of the complexity of $f$ among all monodromies of bundles $N$ which are bundle equivalent to $M$. Using a computer, we can show:

Theorem 1.5. (a) Every mapping class of complexity at most 5 has a power which lies in $\mathcal{C}$. Thus every once-punctured torus bundle of complexity at most 5 is commensurable to one with property $\mathrm{VH}$.

(b) There are 745 once-punctured torus bundles of complexity at most 12 ; with at most 36 exceptions, these are all commensurable to bundles with property $\mathrm{VH}$. 


\section{Idea of Proof}

Our proof of Theorem 1.2 is inspired by the arguments of Cooper and Walsh [4], who show that every fibered knot in a $\mathbb{Z} / 2$ homology sphere admits infinitely many virtually Haken surgeries. The idea is to replace $M$ with a finite cover $\widetilde{M}$ in which the fiber has multiple boundary components. Then one hopes to find a non-separating surface in $\widetilde{M}$ which is not a fiber, so that the techniques of [3] may be applied to find an essential surface in a cyclic cover of $\widetilde{M}$. Finally one must show that certain slopes on $\partial M$ lift to the ultimate cover.

Our argument diverges from that of [4] in that we choose $\widetilde{M}$ to have three or four boundary components instead of two. Since we have two covers to work with, we obtain two surfaces, which is the key to proving property VH. Furthermore, the surfaces which we construct are disjoint from one of the boundary components of $\widetilde{M}$, and thus cannot be fibers. Thus we avoid a number of issues in [4] involving semi-bundle structures.

However, we encounter several new issues. First, it is possible that our surfaces may become fibers after Dehn filling, and to rule this out requires the computation of an Alexander polynomial. Secondly, and more importantly, we require that the boundary components of the non-separating surface in $\widetilde{M}$ must all project to the same slope in $M$. To arrange this, we must develop techniques for constructing surfaces, and computing slopes, explicitly. Some of these techniques (those in Section 3) may be applied to any bundle, but some (those in Section 4) exploit special features of the genus 1 mapping class group.

\section{A Question}

Although some of the methods in this paper apply only to punctured torus bundles, it is conceivable Conjecture 1.1 can be attacked along broadly similar lines. A key step would be to answer the following (presumably difficult) question:

Question: Let $M$ be a knot manifold. Is there a finite cover $\widetilde{M}$ of $M$ which contains a non-separating surface, which is disjoint from some component of $\partial \widetilde{M}$, and whose boundary curves all project to the same slope on $\partial M$ ?

By the results of this paper, the answer to the question is yes if $M$ is a punctured torus bundle.

\section{Plan of paper}

Section 2 fixes a choice of basis for $H_{1}(\partial M)$, when $M$ is any 3-manifold fibering over $S^{1}$. In Section 3, we show how to compute "algebraic" boundary slopes of non-separating surfaces in bundles. In Section 4, we prove the 
main theorem. In Sections 5-7, we give methods for explicit computations of slopes. Section 8 is devoted to the example of the figure eight knot exterior. In Section 9, we prove Theorem 1.4. Finally, in Section 10, we discuss our computer-generated data.

\section{Framing convention}

Let $f: F \rightarrow F$ be an automorphism of a compact, orientable surface, and let $M=F \times[0,1] /(x, 0)=(f x, 1)$ be the 3 -manifold fibering over $S^{1}$ with monodromy $f$. Let $\lambda_{1}, \ldots, \lambda_{k}$ be the boundary components of $F$, and suppose that $f$ acts trivially on $\partial F$, so $M$ has torus boundary components $T_{1}, \ldots, T_{k}$.

We wish to fix a framing for $\partial M$ (see figure 1). For $i=1, \ldots, k$, we fix a point $p_{i} \in \lambda_{i}$, and let the meridian, $\mu_{i} \subset T_{i}$, be the suspension of the point $p_{i}$. The orientation of $\mu_{i}$ is chosen so that the map from $[0,1]$ (with standard orientation) to $\mu_{i}$ given by $t \rightarrow\left(p_{i}, 1-t\right)$ is orientation-preserving. We let the longitude, $\lambda_{i}$, of $T_{i}$ be given by $\lambda_{i} \times\{1\}$. We orient $\lambda_{i}$ so that $I\left(\mu_{i}, \lambda_{i}\right)=1$, where $I(\cdot, \cdot)$ is the standard intersection pairing on $H_{1}(\partial M)$ (see figure 1).

Given a surface $S$ properly embedded in $M$, we may specify the homology classes of the boundary curves of $S$ by a vector $\left(\alpha_{1}, \ldots, \alpha_{k}\right)$, where each $\alpha_{i}$ is an ordered pair of integers. We shall refer to this as the vector of "algebraic boundary slopes" of $S$. For example, if we say that $S$ has algebraic boundary slopes $((1,2),(0,0),(0,3))$, we mean that $\left[S \cap T_{1}\right]=\left[\mu_{1}+2 \lambda_{1}\right]$ in $H_{1}\left(T_{1}\right)$, that $\left[S \cap T_{2}\right]=[0] \in H_{1}\left(T_{2}\right)$ and that $\left[S \cap T_{3}\right]=3\left[\lambda_{3}\right]$ in $H_{1}\left(T_{3}\right)$. Unless otherwise specified, all homology groups in this paper will be taken with $\mathbb{Z}$ coefficients.

\section{Homology and boundary slopes of bundles}

We begin by recalling some well-known facts. If $M$ is a manifold which fibers over $S^{1}$, with monodromy $f: F \rightarrow F$, then there is a corresponding Wang exact sequence

$$
\begin{aligned}
\ldots \longrightarrow H_{j}(M) \stackrel{\theta}{\longrightarrow} H_{j-1}(F) \stackrel{f_{*}-\mathrm{Id}}{\longrightarrow} H_{j-1}(F) \\
\stackrel{i_{*}}{\longrightarrow} H_{j-1}(M) \longrightarrow \ldots,
\end{aligned}
$$

where $\theta$ is the map induced by intersection with $F \times\{0\}$ (see [8]). By a simple modification of the proof, one can also obtain a relative version of this sequence. A direct check shows that the relative Wang sequence 
fits with the exact sequence of a pair into the following commutative diagram:

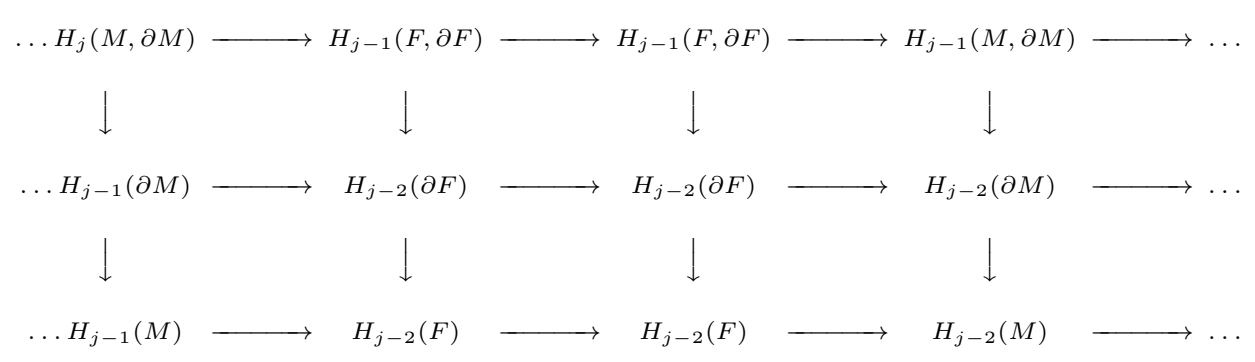

In the case where $M$ is a 3-manifold, one may use the Wang sequence to compute that $\operatorname{Rank} H_{2}(M, \partial M ; \mathbb{Q})=1+\operatorname{Rank}\left(\operatorname{fix}\left(f_{*}\right) ; \mathbb{Q}\right)$, where $\operatorname{fix}\left(f_{*}\right)$ denotes the set of vectors in $H_{1}(F, \partial F)$ which are fixed by $f_{*}$.

Suppose now that $f$ acts trivially on $\partial F$. As described in the previous section, for each component of $\partial M$, there is a canonical meridian, given by the suspension of a point on $\partial F$, and longitude, given by intersection with $F$. This gives a canonical basis for $H_{1}(\partial M)$, and we have corresponding projections $\mu: H_{1}(\partial M) \rightarrow H_{0}(\partial F)$, which sends each longitude to 0 , and $\lambda$ : $H_{1}(\partial M) \rightarrow H_{1}(\partial F)$, which sends each meridian to 0 . We will fix a preferred component $\ell$ of $\partial F$, and let $\pi_{\ell}$ be the projection map from $H_{1}(\partial F)$ to $H_{1}(\ell)$.

We may define a map $\eta: H_{2}(M, \partial M) \rightarrow \operatorname{fix}\left(f_{*}\right) \times H_{1}(\ell)$ by

$$
\eta[R]=\left([R \cap F \times\{0\}], \pi_{\ell} \lambda([\partial R])\right) .
$$

We claim that $\eta$ is injective. Indeed, if $[R] \in \operatorname{ker}(\eta)$, then $R \cap F \times$ $\{0\}$ is trivial in $H_{1}(F, \partial F)$. Therefore, by the relative Wang sequence, $[R] \in i_{*} H_{2}(F, \partial F)$. But since $[R] \in \operatorname{ker}(\eta)$, then $\pi_{\ell}[\partial R]$ is trivial in $H_{1}(\ell)$, and so $[R]=0$, and $\operatorname{ker}(\eta)=0$.

If $[\delta] \in \operatorname{fix}\left(f_{*}\right) \subset H_{1}(F, \partial F)$, then $[f \delta-\delta]=0 \in H_{1}(F, \partial F)$, and so the 1chain $f \delta-\delta$ represents a class $[x]$ in Image $\left(i_{*}: H_{1}(\partial F) \rightarrow H_{1}(F)\right)$. Note $\lambda_{1}, \ldots, \lambda_{k}$ generate a rank $k-1$ subgroup of $H_{1}(F)$, and so $[x]$ is unique, up to adding copies of $[\partial F]$. Thus we may define $\psi$ : fix $\left(f_{*}\right) \times H_{1}(\ell) \rightarrow H_{1}(\partial F)$ uniquely, by requiring

$$
i_{*} \psi([\delta], m[\ell])=[f \delta-\delta], \quad \text { and } \quad \pi_{\ell} \psi([\delta], m[\ell])=m[\ell] .
$$


We have the following diagrams:

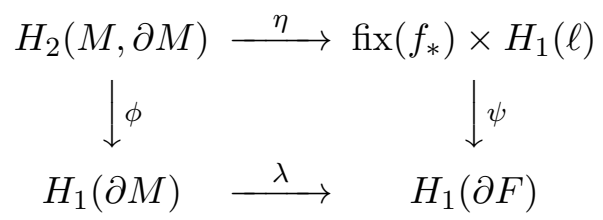

and

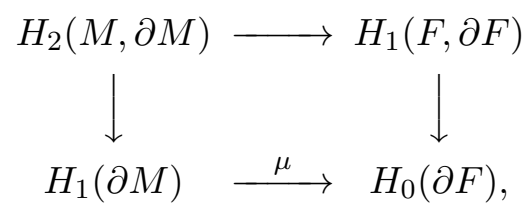

where in the first diagram, $\phi$ is the boundary map, and in the second diagram, all unlabeled maps come from the exact sequence of the pair, and the Wang sequence.

Lemma 3.1. The above diagrams commute.

Proof. For the second diagram, note that the map $\mu$ agrees with the map $\theta$ from the Wang sequence for $\partial M$. Thus the second diagram fits into the larger diagram given at the beginning of the section, which commutes.

For the first diagram, suppose we are given a class $[R] \in H_{2}(M, \partial M)$. A 2-chain homologous to $R$ may be constructed as follows. We let $\delta=R \cap$ $(F \times\{0\})$, and let $\eta[R]=([\delta], m)$. By definition of the map $\psi$, we have $[\delta-f \delta]=-i_{*} \psi([\delta], m)$. Thus there is a map $g: X \rightarrow F$, where $X$ is an orientable surface with $\partial X=\partial_{0} X \amalg \partial_{1} X$, such that $\left.g\right|_{\partial_{0} X}$ is the immersed curve $\delta-f \delta$ and such that $g \partial_{1} X \subset \partial F$, with $i_{*}\left[g \partial_{1} X\right]=i_{*} \psi([\delta], m)$; since $i_{*}[\partial F]=0$, we may also assume that $g \partial_{1} X \cap \ell=\emptyset$. Let $\partial_{00} X \subset \partial_{0} X$ be the union of arcs which map to $\delta$, and let $\partial_{01} X \subset \partial_{0} X$ be the union of arcs which map to $-f \delta$. Let $\sigma$ be a properly embedded collection of separating arcs in $X$, with $\partial \sigma=\partial_{00} X \cap \partial_{01} X$. Let $h: X-\sigma \rightarrow\{0,1\}$ be a continuous map, such that $h\left(\partial_{00} X\right)=0$ and $h\left(\partial_{01} X\right)=1$.

Let $Y$ be an orientable surface obtained from $\overline{X-\sigma} \amalg(\sigma \times[0,1])$, by identifying $\partial_{00} X$ and $\partial_{01} X$ according to the map $f$, and identifying $\sigma \times$ $\partial[0,1]$ with $\overline{X-\sigma}-(X-\sigma)$ in the obvious way.

Then we may construct a map $j: Y \rightarrow M$, by the rule $j(x)=(g x, h x)$, if $x \in X-\sigma$, and $j(x, t)=(g x, t)$ if $(x, t) \in \sigma \times[0,1]$. By construction, $\eta([j Y])=$ $([\delta], 0)$, and so $\eta([j Y]+m[F])=([\delta], m)=\eta[R]$. Thus $[j Y]+m[F]=[R]$. 
Also, by inspection, $\lambda \phi[j Y]=\left[g \partial_{1} X\right]=\psi([\delta], 0)$, and so

$$
\begin{aligned}
\lambda \phi[R] & =\lambda \phi([j Y]+m[F]) \\
& =\psi([\delta], 0)+m[\partial F] \\
& =\psi([\delta], m) \\
& =\psi \eta([j Y]+m[F]) \\
& =\psi \eta[R] .
\end{aligned}
$$

Corollary 3.2. Suppose $R$ is a properly embedded, orientable, nonseparating surface in $M$, and let $\delta=R \cap(F \times\{0\})$. Then the algebraic boundary slopes of $R$ satisfy

$$
\begin{aligned}
& \mu([\partial R])=[\delta \cap \partial F] \in H_{0}(\partial F), \\
& \lambda([\partial R])=[f \delta-\delta]+k[\partial F] \in H_{1}(\partial F) \quad \text { for some integer } k .
\end{aligned}
$$

The following corollary employs notation introduced in Section 2.

Corollary 3.3. Suppose there is an arc $\delta$ properly embedded in $F$, with $[\delta \cap$ $\partial F]=\sum a_{i}\left[p_{i}\right] \in H_{0}(\partial F)$, and $[f \delta-\delta]=\sum b_{i}\left[\lambda_{i}\right] \in H_{1}(F)$. Then there is a non-separating, orientable surface $R$ properly embedded in $M$, with algebraic boundary slopes $\left(\left(a_{1}, b_{1}\right), \ldots,\left(a_{k}, b_{k}\right)\right)$.

Proof. By the Wang exact sequence, there is a class $[R] \in H_{2}(M, \partial M)$, such that $[R \cap F \times\{0\}]=\theta[R]=[\delta] \in H_{1}(F, \partial F)$. Then, by the previous corollary, we have $\mu([\partial R])=[\delta \cap \partial F] \in H_{0}(\partial F)$, and $\lambda([\partial R])=[f \delta-\delta]+$ $k[\partial F] \in H_{1}(\partial F)$. Adding a multiple of $[F]$ to $[R]$, we get $\lambda[\partial R]=[f \delta-\delta]$. Since every class $H_{2}(M, \partial M)$ can be represented by a properly embedded surface in $M$, the corollary follows.

\section{Proof of Theorem 1.2}

We shall make use of the following, which can be proved by straightforward applications of the methods of [3].

Theorem 4.1. For any compact, orientable surface, $S$, there is a positive integer $n=n(S)$, depending only on the topological type of $S$, such that the following is true. Let $M$ be any compact, orientable, irreducible, atoroidal 3-manifold, with two torus boundary components, containing a properly embedded, orientable, incompressible, non-separating surface homeomorphic to $S$, which is not a fiber in a fibration of $M$, with algebraic boundary slopes 
$\left(\delta_{1}, \delta_{2}\right)$, where $\delta_{i} \neq 0$. Then, if $\left|I\left(\alpha_{1}, \delta_{1}\right)\right|=\left|I\left(\alpha_{2}, \delta_{2}\right)\right|>n(S)$, the manifold obtained by Dehn filling $M$ along $\alpha_{1}$ and $\alpha_{2}$ is virtually Haken.

Remark. By choosing the function $n(S)$ appropriately, we may assume that, whenever $S^{\prime}$ is obtained by compressing $S$, we have $n\left(S^{\prime}\right)<n(S)$.

We are now ready to prove the main theorem.

Proof of Theorem 1.2. Recall that the kernel of $\phi: \mathcal{M}_{1}^{1} \rightarrow S L_{2}(\mathbb{Z})$ is generated by a Dehn twist along a peripheral curve in $F$. It follows that if $f, g \in \mathcal{M}_{1}^{1}$, and $f_{*}=g_{*}$, then $M_{f}$ is bundle equivalent to $M_{g}$. Thus we may reduce to the case where $f \in J_{i}=\left\langle D_{y}^{i}, D_{x}\right\rangle$.

Case (a) $f \in J_{3}$. In this case, $f$ can be written as a word $W$ in $D_{x}$ and $D_{y}^{3}$. Let $m=m_{3}$ denote the exponent sum of $D_{x}$ in $W$. Let $\beta=\beta_{3}^{1}$ be the slope $(3, m) / \operatorname{gcd}(3, m)$ on $\partial M$.

Let $\theta: \widehat{F} \rightarrow F$ be the 3 -fold cyclic cover of $F$ dual to the curve $x$. Since $f \in H_{3}$, then $f$ lifts to an automorphism $\widehat{f}: \widehat{F} \rightarrow \widehat{F}$ which acts trivially on $\partial \widehat{F}$. Let $\pi: \widehat{M} \rightarrow M$ be the 3 -fold cover of $M$ induced by the lift $\widehat{f}: \widehat{F} \rightarrow \widehat{F}$.

If $g$ is a map between two surfaces with boundary, we let $g_{\sharp}$ be the induced map on $H_{1}$ rel. boundary. Let $\lambda_{1}, \lambda_{2}, \lambda_{3}$ be the components of $\partial \widehat{F}$ (with pre-image orientations induced from $\lambda$ ), and let $p_{i} \in \lambda_{i}$ be the preimages of $p \in \lambda$. Let $\delta_{i}$ be an arc connecting $\lambda_{i}$ and $\lambda_{i+1}$, oriented to point toward $\lambda_{i}$, as pictured in figure $2(\mathrm{a})$. Let $\delta=\delta_{1}-\delta_{2}$. Then it is easy to see that $[\delta]$ is a non-zero class in $H_{1}(\widehat{F}, \partial \widehat{F})$ which is fixed by the lifts of $D_{x}$ and $\left(D_{y}\right)^{3}$, and therefore by $\widehat{f}_{\sharp}$. Moreover, $[f \delta-\delta]=-m\left[\lambda_{2}\right] \in H_{1}(F)$.

Let $\tau$ be the covering transformation of $\widehat{F}$ such that $\tau\left(\delta_{1}\right)=\delta_{2}$.

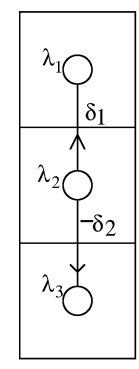

a

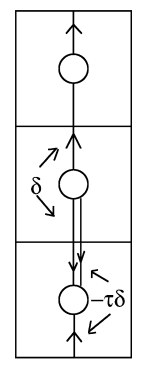

b

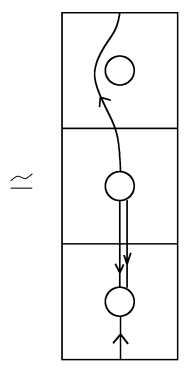

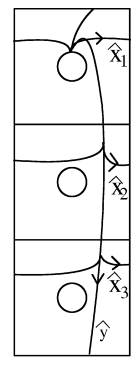

C

Figure 2: (a) A class in $\operatorname{ker}\left(p_{*}\right)$. (b) Fixed class $[\delta-\tau \delta]$ in $H_{1}(\widehat{F}, \partial \widetilde{F})$, corresponding to the surface $S$. (c) Generators for $\pi_{1} \widehat{F}$. 
Lemma 4.2. There is a non-separating surface $R \subset \widehat{M}$ whose boundary slopes are given by $((0,0),(-3,-m),(3, m))$.

Proof. We have $[f(\delta-\tau \delta)-(\delta-\tau \delta)]=m\left[\lambda_{3}\right]-m\left[\lambda_{2}\right] \in H_{1}(F)$, and $[(\delta-$ $\tau \delta) \cap \partial F]=0\left[p_{1}\right]-3\left[p_{2}\right]+3\left[p_{3}\right] \in H_{0}(\partial F)$. The lemma now follows from Corollary 3.3.

Let $T_{i}$ be the component of $\partial \widehat{M}$ containing $p_{i}$. By attaching annuli to $R$, we may assume that $R$ is disjoint from $T_{1}$. Let $\alpha$ be a slope on $\partial M$, let $\widehat{\alpha}_{i}$ be the lift of $\alpha$ to $T_{i}$, so $\widehat{M}\left(\widehat{\alpha}_{1}\right)$ is a manifold with two torus boundary components, and let $S$ be an incompressible surface in $\widehat{M}\left(\widehat{\alpha}_{1}\right)$ obtained by compressing $R$. Then $S$ has boundary slopes which project to curves of slope $\beta_{3}^{1}$ in $\partial M$.

We wish to show that $S$ is typically not a fiber, and to do so requires the computation of an Alexander polynomial. Let $N$ be the infinite cyclic cover of $\widehat{M}\left(\widehat{\alpha}_{1}\right)$ which is dual to $S$, and let $\Delta(s)$ be the Alexander polynomial associated to this infinite cyclic cover.

Let $\theta: \widetilde{F} \rightarrow \widehat{F}$ be the infinite cyclic cover dual to $\delta-\tau \delta$. We let $\widehat{x}_{i}=y^{i-1} x y^{-(i-1)} \in \pi_{1} \widehat{F}$, and let $\widehat{y}=y^{3} \in \pi_{1} \widehat{F}$ (all based at $p_{1}$ ). Let $\tilde{p_{1}} \in \theta^{-1} p_{1}$ be a basepoint for $\widetilde{F}$. There are two possible choices for $s$; we choose $s$ so there is a lift of $\widehat{x}_{1}$ to $\widetilde{F}$, with boundary $-p_{1}+s p_{1}$.

Let $\widetilde{f}$ be the lift of $f$ to $\widetilde{F}$ which fixes $\theta^{-1} p$ pointwise. Then $\tilde{f}$ acts on the module $H_{1}\left(\widetilde{F}, \theta^{-1} p_{1}\right)$ by an automorphism $f_{*}$, and since $\widetilde{f}$ fixes $\theta^{-1} p_{1}$ pointwise, then $\operatorname{Im}\left(\operatorname{Id}-f_{*}\right) \subset H_{1}(\widetilde{F})$. The map Id $-f_{*}$ has a kernel containing $\left\langle\left[\widehat{x}_{1} \widehat{x}_{2}^{-1}\right]\right\rangle$ (we sometimes use the same symbol for a loop in $\widehat{F}$ based at $p_{1}$, and its lift to $\widetilde{F}$ based at $\left.\underset{\sim}{\tilde{p}_{1}}\right)$, and $\underset{\widetilde{F}}{\widetilde{F}}$ there is an induced map $\overline{\mathrm{Id}-f_{*}}: H_{1}\left(\widetilde{F}, \theta^{-1} p_{1}\right) /\left\langle\left[\widehat{x}_{1} \widehat{x}_{2}^{-1}\right]\right\rangle \rightarrow H_{1}(\widetilde{F}) \subset H_{1}\left(\widetilde{F}, \theta^{-1} p_{1}\right)$.

There is a $\mathbb{Z}\left[s^{ \pm 1}\right]$-module decomposition $H_{1}\left(\widetilde{F}, \theta^{-1} p_{1}\right) \cong H_{1}(\widetilde{F}) \oplus\left\langle\left[\widehat{x}_{1}\right]\right\rangle$, coming from the exact sequence for the pair $\left(\widetilde{F}, \theta^{-1} p_{1}\right)$. In fact, it is a free $\mathbb{Z}\left[s^{ \pm 1}\right]$-module, with basis

$$
\mathcal{B}=\left([\widehat{y}],\left[\widehat{x}_{1} \widehat{x}_{2}^{-1}\right],\left[\widehat{x}_{1}^{2} \widehat{x}_{3}\right],\left[\widehat{x}_{1}\right]\right)
$$

obtained by lifting a basis for the kernel of the corresponding map $H_{1}(\widehat{F}) \rightarrow$ $\mathbb{Z}$, and then adding $\left[\widehat{x}_{1}\right]$.

We let $\left[f_{*}\right]$ be the matrix representative for $f_{*}$ with respect to $\mathcal{B}$. Then $\overline{\mathrm{Id}-f_{*}}$ can be represented by a matrix $\left[\overline{\mathrm{Id}-f_{*}}\right]$, which is obtained by deleting the second column and fourth row of $\mathrm{Id}-\left[f_{*}\right]$. 
Lemma 4.3. (a) The matrix $\left[f_{*}\right]$ is given by matrix $W\left(\left[D_{x *}\right],\left[D_{y *}^{3}\right]\right)$, where

$$
\left[D_{x *}\right]=\left(\begin{array}{cccc}
s & 0 & 0 & 0 \\
s^{-1} & 1 & s-s^{-1} & 0 \\
-s^{-1} & 0 & s^{-1} & 0 \\
0 & 0 & 0 & 1
\end{array}\right)
$$

and

$$
\left[D_{y *}^{3}\right]=\left(\begin{array}{cccc}
1 & 0 & 1+s+s^{2} & 1 \\
0 & 1 & 0 & 0 \\
0 & 0 & 1 & 0 \\
0 & 0 & 0 & 1
\end{array}\right)
$$

(b) The Alexander polynomial $\Delta(s)$ is the determinant of the following matrix:

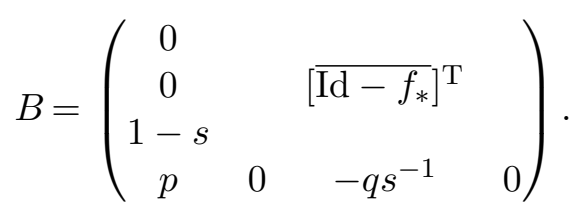

Using Lemma 4.3 and a computer, the Alexander polynomial $\Delta(s)$ can be easily computed. We note that Lemma 4.3 (a) will not be used until the section on computer calculations.

Proof. Part (a) is a computation, which can be done with Fox derivatives. We leave this to the reader.

For part (b) let $\widetilde{M}$ be the $\mathbb{Z}$-cover of $\widehat{M}$ which is dual to $R$. We begin by computing a presentation for $H_{1}(\widetilde{M})$ as a $\mathbb{Z}\left[s^{ \pm 1}\right]$-module. Then $\Delta(s)$ will be the determinant of the corresponding relation matrix.

We choose the point $p_{1}$ as a basepoint for $\pi_{1} \widehat{M}$. We have $\pi_{1} \widehat{F} \subset \pi_{1} \widehat{M}$, and we let $\widehat{t} \in \pi_{1} \widehat{M}$ be the lift of the longitude $\mu$ pictured in figure 1 . We have the following presentation for $\pi_{1}(\widehat{M})$ (the relators are written in nonreduced form for future convenience):

$$
\begin{aligned}
\pi_{1} \widehat{M} & =\left\langle\widehat{y}, \widehat{x}_{1}, \widehat{x}_{1} \widehat{x}_{2}^{-1}, \widehat{x}_{1}^{2} \widehat{x}_{3}, \widehat{t} \mid R_{1}, R_{2}, R_{3}, R_{4}\right\rangle \\
R_{1} & =\widehat{t} \widehat{y} \widehat{t}^{-1} \widehat{y}^{-1}\left(\widehat{y} \widehat{f}_{*} \widehat{y}^{-1}\right) \\
R_{2} & =\widehat{t} \widehat{x}_{1} \widehat{t}^{-1} \widehat{x}_{1}^{-1}\left(\widehat{x}_{1} \widehat{f}_{*} \widehat{x}_{1}^{-1}\right), \\
R_{3} & =\widehat{t} \widehat{x}_{1} \widehat{x}_{2}^{-1} \widehat{t}^{-1}\left(\widehat{x}_{1} \widehat{x}_{2}^{-1}\right)^{-1}\left(\widehat{x}_{1} \widehat{x}_{2}^{-1} \widehat{f}_{*}\left(\widehat{x}_{1} \widehat{x}_{2}^{-1}\right)^{-1}\right), \\
R_{4} & =\widehat{t} \widehat{x}_{1}^{2} \widehat{x}_{3} \widehat{t}^{-1}\left(\widehat{x}_{1}^{2} \widehat{x}_{3}\right)^{-1}\left(\widehat{x}_{1}^{2} \widehat{x}_{3} \widehat{f}_{*}\left(\widehat{x}_{1}^{2} \widehat{x}_{3}\right)^{-1}\right)
\end{aligned}
$$


The group $H_{1}(\widetilde{M})$ also has the structure of a $\mathbb{Z}\left[s^{ \pm 1}\right]$-module, where again $s$ is chosen so that the lift of $\widehat{x}_{1}$ to $\widetilde{M}$ based at $\tilde{p}_{1}$ has boundary $-\tilde{p}_{1}+s \tilde{p}_{1}$. Then, as a $\mathbb{Z}\left[s^{ \pm 1}\right]$-module, $H_{1}(\widetilde{M})$ has an ordered generating set $\mathcal{B}^{\prime}=\left([\widehat{t}],[\widehat{y}],\left[\widehat{x}_{1} \widehat{x}_{2}^{-1}\right],\left[\widehat{x}_{1}^{2} \widehat{x}_{3}\right]\right)$.

The relators of $H_{1}(\widetilde{M})$ (as a $\mathbb{Z}\left[s^{ \pm 1}\right]$-module) may be obtained from the relators $R_{1}, \ldots, R_{4}$ of $\pi_{1} \widehat{M}$. We have:

$$
\begin{aligned}
R_{1} & =\widehat{t} \widehat{t}^{-1} \widehat{y}^{-1}\left(\widehat{y} \widehat{f}_{*} \widehat{y}^{-1}\right) \\
& \Rightarrow\left[\widehat{y} \widehat{f}_{*} \widehat{y}^{-1}\right]=0 \\
R_{2} & =\widehat{t} \widehat{x}_{1} \widehat{t}^{-1} \widehat{x}_{1}^{-1}\left(\widehat{x}_{1} \widehat{f}_{*} \widehat{x}_{1}^{-1}\right) \\
& \rightarrow(1-s)[\widehat{t}]+\left[\widehat{x}_{1} \widehat{f}_{*} \widehat{x}_{1}^{-1}\right]=0 \\
R_{3} & =\widehat{t}\left(\widehat{x}_{1} \widehat{x}_{2}^{-1}\right) \widehat{t}^{-1}\left(\widehat{f}_{*} \widehat{x}_{1} \widehat{x}_{2}^{-1}\right)^{-1} \\
& =\widehat{t}\left(\widehat{x}_{1} \widehat{x}_{2}^{-1}\right) \widehat{t}^{-1}\left(\widehat{x}_{1} \widehat{x}_{2}^{-1}\right)^{-1}\left(\text { since } x_{1} x_{2}^{-1}\right.
\end{aligned}
$$

is homologous to a boundary component)

$$
\Rightarrow 0=0
$$$$
R_{4}=\widehat{t}\left(\widehat{x}_{1}^{2} \widehat{x}_{3}\right) \widehat{t}^{-1}\left(\widehat{x}_{1}^{2} \widehat{x}_{3}\right)^{-1}\left(\left(\widehat{x}_{1}^{2} \widehat{x}_{3}\right) \widehat{f}_{*}\left(\widehat{x}_{1}^{2} \widehat{x}_{3}\right)^{-1}\right)
$$$$
\Rightarrow\left[\left(\widehat{x}_{1}^{2} \widehat{x}_{3}\right) \widehat{f}_{*}\left(\widehat{x}_{1}^{2} \widehat{x}_{3}\right)^{-1}\right]=0 .
$$

Thus a presentation matrix for $H_{1}(\widetilde{M})$, in terms of $\mathcal{B}^{\prime}$, is given by:

$$
A=\left(\begin{array}{cc}
0 & \\
0 & {\overline{\mathrm{Id}-f_{*}}}^{\mathrm{T}} \\
1-s &
\end{array}\right) .
$$

The presentation matrix for $H_{1}(N)$ is obtained from $A$ by adding a single relator of the form $\left[t^{p}\left(x^{-1} y x y^{-1}\right)^{q}\right]=0$, where $p, q \in \mathbb{Z}$. This yields the relator $p[\widehat{t}]-q s^{-1}\left[x_{1} x_{2}^{-1}\right]=0$. Thus the presentation matrix for $H_{1}(N)$ is:

$$
\left(\begin{array}{cccc}
0 & & \\
0 & {\overline{\mathrm{Id}-f_{*}}}^{\mathrm{T}} & \\
1-s & & & \\
p & 0 & -q s^{-1} & 0
\end{array}\right)
$$

and so $\Delta(s)$ is the determinant of this matrix. 
Corollary 4.4. There is a slope $\beta_{3}^{2}$ such that, if $I\left(\alpha, \beta_{3}^{2}\right)>1$, then $S$ is not a fiber in a fibration of $\widehat{M}\left(\widehat{\alpha}_{1}\right)$.

Proof. It is well known that, if an infinite cyclic cover of a compact 3 -manifold is dual to a fiber in a fibration, then the corresponding Alexander polynomial is monic. By Lemma $4.3(\mathrm{~b}), \Delta(s)=p \operatorname{Det} M_{1}+q \operatorname{Det} M_{2}$ for some matrices $M_{1}$ and $M_{2}$ with entries in $\mathbb{Z}\left[s^{ \pm 1}\right]$. Thus the leading term of $\Delta(s)$ is $p n_{1}+q n_{2}$, for some integers $n_{1}, n_{2}$. We let $\beta_{3}^{2}=\left(-n_{2}, n_{1}\right) / \operatorname{gcd}\left(n_{2}, n_{1}\right)$. Then if $\left|I\left(\alpha, \beta_{3}^{2}\right)\right| \neq 1, \Delta(s)$ is non-monic.

Let $n$ be a positive integer. By Thurston's hyperbolic Dehn surgery theorem, we may assume that $n$ is chosen large enough so that $\widehat{M}\left(\widehat{\alpha}_{1}\right)$ is hyperbolic whenever $I\left(\alpha, \beta_{3}^{1}\right)>n$. We also assume that $n$ is larger than the integer $n(R)$ given by Theorem 4.1, and hence also bigger than $n(S)$.

Suppose $\left|I\left(\alpha, \beta_{3}^{1}\right)\right|=k>n$, and that $\left|I\left(\alpha, \beta_{3}^{2}\right)\right|>1$. Since $k>n$, then $\widehat{M}\left(\widehat{\alpha}_{1}\right)$ is hyperbolic, and since $\left|I\left(\alpha, \beta_{3}^{2}\right)\right|>1$ then, by Corollary $4.4, S$ is not a fiber in a fibration of $\widehat{M}\left(\widehat{\alpha}_{1}\right)$. Let $\widehat{\alpha}_{2}, \widehat{\alpha}_{3}$ be the lifts of $\alpha$ to the components of $\widehat{M}\left(\widehat{\alpha}_{1}\right)$. Then $\left|I\left(\widehat{\alpha}_{2}, \partial S\right)\right|=\left|I\left(\widehat{\alpha}_{3}, \partial S\right)\right|=k>n \geq n(S)$, so by Theorem 4.1, the Dehn filling of $\widehat{M}\left(\widehat{\alpha}_{1}\right)$ along $\widehat{\alpha}_{2}$ and $\widehat{\alpha}_{3}$ is virtually Haken. Since this manifold covers $M(\alpha)$, then $M(\alpha)$ is virtually Haken.

Case (b): $f \in J_{4}$. The argument is similar to the argument for Case (a) Let $\beta=\beta_{4}^{1}=(2, m) / \operatorname{gcd}(2, m)$, where $m=m_{4}$ is the exponent sum of $D_{x}$ in the word $W\left(D_{x}, D_{y}^{4}\right)$ which represents $f$. We let $\widehat{F}$ be the 4 -fold cyclic cover dual to $x$, and let $\widehat{M}$ be the corresponding cover of $M$. We fix a basepoint $p_{1} \in \partial \widehat{M}$, and let $p_{i}$ be the translate of $p_{1}$ by the covering translation corresponding to $y^{i-1}$. We let $T_{i}$ be the component of $\partial \widehat{M}$ containing $p_{i}$.

We define arcs $\delta_{1}$ and $\delta_{2}$ in $\widehat{F}$ as pictured in figure 3 , and let $\delta=\delta_{1}-\delta_{2}$. In this case, the non-separating surface $R$ corresponding to $[\delta]-\left[\tau^{2} \delta\right]$ has boundary slopes $((0,0),(-2,-m),(0,0),(2, m)) \neq((0,0),(0,0),(0,0),(0,0))$. By compressing $R$, we obtain a properly embedded, orientable, nonseparating, incompressible surface $S$ in $\widehat{M}\left(\widehat{\alpha}_{1}, \widehat{\alpha}_{3}\right)$, whose boundary curves all project to curves of slope $\beta$ on $\partial M$.

Let $N$ be the infinite cyclic cover of $\widehat{M}\left(\widehat{\alpha}_{1}, \widehat{\alpha}_{3}\right)$ dual to $S$, and let $\Delta(s)$ be the corresponding Alexander polynomial. Let $\theta: \widetilde{F} \rightarrow \widehat{F}$ be the infinite cyclic cover of $\widehat{F}$ dual to $\delta-\tau^{2} \delta$. We let $\widehat{x}_{i}=y^{i-1} x y^{-(i-1)} \in \widehat{F}$, and let $\widehat{y}=y^{4} \in \widehat{F}$. 


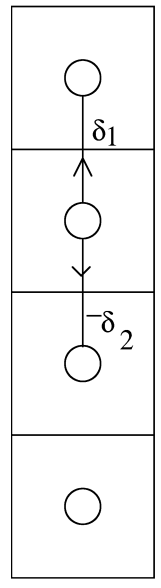

a

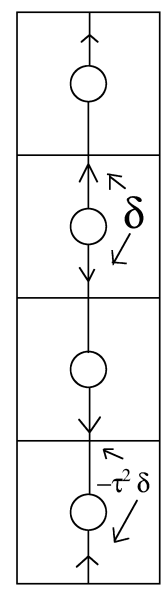

b

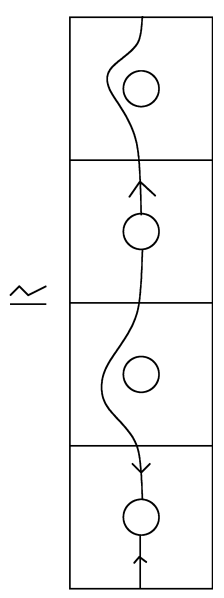

Figure 3: (a) A class in $\operatorname{ker}\left(p_{*}\right)$. (b) A fixed class in $H_{1}(\widehat{F}, \partial \widetilde{F})$, corresponding to the surface $S$.

The automorphism $\widehat{f}$ induces an automorphism $f_{*}$ of $H_{1}\left(\widetilde{F}, \theta^{-1} p_{1} \cup p_{3}\right)$. There is an induced map

$$
\begin{gathered}
\overline{\operatorname{Id}-f_{*}}: H_{1}\left(\widetilde{F}, \theta^{-1}\left(p_{1} \cup p_{3}\right)\right) /\left\langle\left[\widehat{x}_{1} \widehat{x}_{2}^{-1}\right],\left[\widehat{x}_{3} \widehat{x}_{4}^{-1}\right]\right\rangle \\
\longrightarrow H_{1}(\widetilde{F}) \subset H_{1}\left(\widetilde{F}, \theta^{-1}\left(p_{1} \cup p_{3}\right)\right) .
\end{gathered}
$$

The $\mathbb{Z}\left[s^{ \pm 1}\right]$-module $H_{1}\left(\widetilde{F}, \theta^{-1}\left(p_{1} \cup p_{3}\right)\right)$ is free, with basis

$$
\mathcal{B}=\left(\left[\widehat{y}^{4}\right],\left[\widehat{x}_{1} \widehat{x}_{3}\right],\left[\widehat{x}_{1}\right],\left[\widehat{y}^{2}\right],\left[\widehat{x}_{1} \widehat{x}_{2}^{-1}\right],\left[\widehat{x}_{3} \widehat{x}_{4}^{-1}\right]\right) .
$$

We let $\left[f_{*}\right]$ be the matrix representing $f_{*}$, in terms of the basis $\mathcal{B}$. Then $\overline{\mathrm{Id}-f_{*}}$ is represented by a matrix $\left[\overline{\mathrm{Id}-f_{*}}\right]$ obtained from Id $-\left[f_{*}\right]$ by deleting the 5 th and 6 th columns, and the 3 rd and 4th rows.

Lemma 4.5. (a) The matrix $\left[f_{*}\right]$ is given by $W\left(\left[D_{x *}\right],\left[D_{y *}^{4}\right]\right)$, where

$$
\left[D_{x *}\right]=\left(\begin{array}{cccccc}
s & 0 & 0 & 0 & 0 & 0 \\
-\left(1+s^{-1}\right) & s^{-1} & 0 & -s^{-1} & 0 & 0 \\
0 & 0 & 1 & 0 & 0 & 0 \\
0 & 0 & 0 & 1 & 0 & 0 \\
s^{-1} & 1-s^{-1} & 0 & s^{-1} & 1 & 0 \\
0 & 0 & 0 & 0 & 0 & 1
\end{array}\right)
$$


and

$$
\left[D_{y *}^{4}\right]=\left(\begin{array}{cccccc}
1 & 1+s & 1 & 0 & 0 & 0 \\
0 & 1 & 0 & 0 & 0 & 0 \\
0 & 0 & 1 & 0 & 0 & 0 \\
0 & 0 & 0 & 1 & 0 & 0 \\
0 & 0 & 0 & 0 & 1 & 0 \\
0 & 0 & 0 & 0 & 0 & 1
\end{array}\right)
$$

(b) The Alexander polynomial $\Delta(s)$ is the determinant of the following matrix:

$$
\begin{aligned}
& B=\left(\begin{array}{ccccc}
0 & 0 & 0 & 0 & 0 \\
0 & 0 & 0 & 0 & 0 \\
1-s & 0 & 0 & 0 & 0 \\
p & 0 & 0 & 0 & -q s \\
p & 0 & 0 & -q s^{-1} & 0
\end{array}\right) \\
& +\left(\begin{array}{ccccc}
0 & & & \\
0 & & {\overline{\mathrm{Id}-f_{*}}}^{\mathrm{T}} & & \\
0 & & & & \\
0 & & & & \\
0 & 0 & 0 & 0 & 0
\end{array}\right) \cdot\left(\begin{array}{ccccc}
1 & 0 & 0 & 0 & 0 \\
0 & 1 & 0 & 0 & 0 \\
0 & 0 & 1 & 0 & 0 \\
0 & 0 & 0 & p & 0 \\
0 & 0 & 0 & 0 & 1
\end{array}\right),
\end{aligned}
$$

and $\Delta(s)$ is divisible by $q$.

Proof. The proof of part (a) is an elementary application of Fox calculus. In fact, all that is important for our applications is the last row of these matrices.

For part (b), let $\widetilde{M}$ be the infinite cyclic cover of $\widetilde{M}$ dual to $S$. We choose an ordered generating set $\mathcal{B}^{\prime}=\left(\widehat{t}, \widehat{y}^{4}, \widehat{x}_{1} \widehat{x}_{3}, \widehat{x}_{1} \widehat{x}_{2}^{-1}, \widehat{x}_{3} \widehat{x}_{4}^{-1}\right)$ for the $\mathbb{Z}\left[s^{ \pm 1}\right]$ module $H_{1}(\widetilde{M})$.

The relators for $\pi_{1} \widehat{M}$ give the following relations for $H_{1}(\widetilde{M})$ :

$$
\begin{aligned}
R_{1} & =\widehat{t} \widehat{y}^{4} \widehat{t}^{-1} \widehat{y}^{-4}\left(\widehat{y}^{4} \widehat{f}_{*} \widehat{y}^{-4}\right) \\
& \Rightarrow\left[\widehat{y}^{4} \widehat{f}_{*} \widehat{y}^{-4}\right]=0 \\
R_{2} & =\widehat{t} \widehat{x}_{1} \widehat{x}_{2}^{-1} \widehat{t}^{-1}\left(\widehat{x}_{1} \widehat{x}_{2}^{-1}\right)^{-1}\left(\widehat{x}_{1} \widehat{x}_{2}^{-1} \widehat{f}_{*}\left(\widehat{x}_{1} \widehat{x}_{2}^{-1}\right)^{-1}\right) \\
& \Rightarrow 0=0 \\
R_{3} & =\widehat{t} \widehat{x}_{1} \widehat{x}_{3} \widehat{t}^{-1}\left(\widehat{x}_{1} \widehat{x}_{3}\right)^{-1}\left(\widehat{x}_{1} \widehat{x}_{3} \widehat{f}_{*}\left(\widehat{x}_{1} \widehat{x}_{3}\right)^{-1}\right) \\
& \Rightarrow\left[\widehat{x}_{1} \widehat{x}_{3} \widehat{f}_{*}\left(\widehat{x}_{1} \widehat{x}_{3}\right)^{-1}\right]=0
\end{aligned}
$$




$$
\begin{aligned}
R_{4} & =\widehat{t} \widehat{x}_{1} \widehat{t}^{-1} \widehat{x}_{1}^{-1}\left(\widehat{x}_{1} \widehat{f}_{*} \widehat{x}_{1}^{-1}\right) \\
& \rightarrow(1-s)[\widehat{t}]+\left[\widehat{x}_{1} \widehat{f}_{*} \widehat{x}_{1}^{-1}\right]=0 \\
R_{5} & =\widehat{t}_{3} \widehat{x}_{4}^{-1} \widehat{t}^{-1}\left(\widehat{x}_{3} \widehat{x}_{4}^{-1}\right)\left(\widehat{x}_{3} \widehat{x}_{4}^{-1} \widehat{f}_{*}\left(\widehat{x}_{3} \widehat{x}_{4}^{-1}\right)^{-1}\right) \\
& \Rightarrow 0=0 .
\end{aligned}
$$

The module $H_{1}(N)$ has two additional relators, $R_{6}:\left[t^{p}\left(x^{-1} y x y^{-1}\right)^{q}\right]=0$, and $R_{7}:\left[y^{2} t^{p} y^{-2} y^{2}\left(x^{-1} y x y^{-1}\right)^{q} y^{-2}\right]=0$. The relator $R_{6}$ can be written as $p[\widehat{t}]-q s^{-1}\left[\widehat{x}_{1} \widehat{x}_{2}^{-1}\right]=0$. For $R_{7}$, we have

$$
\begin{aligned}
0 & =p\left[y^{2} t y^{-2}\right]+q\left[y^{2}\left(x^{-1} y x y^{-1}\right) y^{-2}\right] \\
& =p\left[\left(y^{2} t y^{-2} t^{-1}\right) t\right]-q s\left[x_{1} x_{2}^{-1}\right] \\
& =p[\widehat{t}]+p\left[y^{2} f_{*} y^{-2}\right]-q s\left[x_{1} x_{2}^{-1}\right] \\
& =p[\hat{t}]+p\left[\overline{\mathrm{Id}-f_{*}} y^{2}\right]-q s\left[x_{1} x_{2}^{-1}\right] .
\end{aligned}
$$

Thus the presentation matrix for $H_{1}(N)$, with respect to $\mathcal{B}$, is

$$
\begin{gathered}
\left(\begin{array}{ccccc}
0 & 0 & 0 & 0 & 0 \\
0 & 0 & 0 & 0 & 0 \\
1-s & 0 & 0 & 0 & 0 \\
p & 0 & 0 & 0 & -q s \\
p & 0 & 0 & -q s^{-1} & 0
\end{array}\right) \\
+\left(\begin{array}{ccccc}
0 & {\overline{\mathrm{Id}}-f_{*}}^{\mathrm{T}} & \\
0 & & \\
0 & & \\
0 & & & \\
0 & 0 & 0 & 0 & 0
\end{array}\right) \cdot\left(\begin{array}{ccccc}
1 & 0 & 0 & 0 & 0 \\
0 & 1 & 0 & 0 & 0 \\
0 & 0 & 1 & 0 & 0 \\
0 & 0 & 0 & p & 0 \\
0 & 0 & 0 & 0 & 1
\end{array}\right) .
\end{gathered}
$$

We then observe that the last column of ${\overline{\mathrm{Id}-f_{*}}}^{\mathrm{T}}$ is all 0s. Therfore the last column of the presentation matrix for $H_{1}(N)$ has a single non-zero entry, namely $-q s$. Thus $\Delta(s)$ is divisible by $q$.

Corollary 4.6. Let $\beta_{4}^{2}=(1,0)$. If $I\left(\alpha, \beta_{4}^{2}\right)>1$, then $S$ is not a fiber in a fibration of $\widehat{M}\left(\widehat{\alpha}_{1}, \widehat{\alpha}_{3}\right)$.

Proof. By Lemma 4.5, $\Delta(s)$ is divisible by $q$. If $|q|=|I(\alpha,(1,0))|>1$, then $\Delta(s)$ is non-monic. 
Now, as in Case (a), an application of Theorem 4.1 shows that $\widehat{M}\left(\widehat{\alpha}_{1}, \widehat{\alpha}_{2}\right.$, $\left.\widehat{\alpha}_{3}, \widehat{\alpha}_{4}\right)$ has a Haken cyclic cover, provided that $\left|I\left(\alpha, \beta_{4}^{2}\right)\right|>1$ and $\left|I\left(\alpha, \beta_{4}^{1}\right)\right|$ is large enough.

\section{Computations: framings}

In the proof of Theorem 1.2, we used the fact that if $f, g \in \mathcal{M}_{1}^{1}$, and $f_{*}=$ $g_{*} \in \mathrm{SL}_{2}(\mathbb{Z})$, then $M_{f}$ is bundle equivalent to $M_{g}$. In this section, we shall show how to compute the effect of this equivalence on the framings.

Let $D_{\lambda}$ be a Dehn twist about a peripheral curve in $F$. Suppose $f \in \mathcal{M}_{1}^{1}$, and that $f_{*}=\mathrm{Id}$. Then $f$ is equivalent in $\mathcal{M}_{1}^{1}$ to $D_{\lambda}^{n}$ for some integer $n$. We define the twist of $f$ by the formula $t(f)=n$.

Lemma 5.1. Suppose that, $f, g \in \mathcal{M}_{1}^{1}$, with $f_{*}=g_{*}$. Then there is a homeomorphism $h: M_{f} \rightarrow M_{g}$, such that, with respect to the standard framings on $\partial M_{f}$ and $\partial M_{g}, h(1,0)=\left(1, t\left(f g^{-1}\right)\right)$ and $h(0,1)=(0,1)$.

Proof. Since $f g^{-1}=D_{\lambda}^{t\left(f g^{-1}\right)}$, then $f g^{-1}$ is isotopic to the identity, by an isotopy which twists $t\left(f g^{-1}\right)$ times around the boundary of $F$. Using this isotopy, one may construct a bundle equivalence between $M_{f}$ and $M_{g}$, and verify that the effect on the framings is as claimed.

Thus, given elements $f, g \in \mathcal{M}_{1}^{1}$, in terms of $D_{x}$ and $D_{y}$, with $f_{*}=g_{*}$, we require a method for computing the twist $t\left(f g^{-1}\right)$.

Lemma 5.2. Let $f, g \in \mathcal{M}_{1}^{1}$ be given as words $W_{f}, W_{g}$ in $D_{x}$ and $D_{y}$, and suppose that $f_{*}=g_{*}$. Then $t\left(f g^{-1}\right)$ is equal to $1 / 12$ of the total sum of the exponents of $D_{x}$ and $D_{y}$ in the word $f g^{-1}=W_{f}\left(D_{x}, D_{y}\right) W_{g}^{-1}\left(D_{x}, D_{y}\right)$.

Proof. We use the following well-known presentation for $\mathrm{SL}_{2}(\mathbb{Z})$ :

$$
\mathrm{SL}_{2}(\mathbb{Z}) \cong\left\langle a, b, \tau \mid \tau=(a b)^{3}=(a b a)^{2}, \tau^{2}=[\tau, a]=[\tau, b]=i d\right\rangle,
$$

where the map sending $D_{x *}$ to $a$ and $D_{y *}$ to $b$ is an isomorphism. Thus if $f_{*}=g_{*}$, then $f g^{-1}=W\left(D_{x}, D_{y}\right)$ is a product of conjugates of the elements $\quad R_{1}=\left(D_{x} D_{y}\right)^{3}\left(D_{x} D_{y} D_{x}\right)^{-2}, \quad R_{2}=\left(D_{x} D_{y}\right)^{3} D_{x}\left(D_{x} D_{y}\right)^{-3} D_{x}^{-1}$, $R_{3}=\left(D_{x} D_{y}\right)^{3} D_{y}\left(D_{x} D_{y}\right)^{-3} D_{y}^{-1}$ and $R_{4}=\left(D_{x} D_{y}\right)^{6}$. By computing the effect of these automorphisms on $\pi_{1} F$, one may check directly that each one is trivial in $\mathcal{M}_{1}^{1}$ except for $R_{4}$, and that $t\left(R_{4}\right)=1$. Therefore $t\left(f g^{-1}\right)$ is equal to the (signed) number of conjugates of $R_{4}$ in $W_{f}\left(D_{x}, D_{y}\right) W_{g}^{-1}\left(D_{x}, D_{y}\right)$. Since the sum of the exponents is 12 on $R_{4}$, and zero on each of $R_{1}, R_{2}$ and $R_{3}$, 
then we see that $t\left(f g^{-1}\right)$ is simply $1 / 12$ of the total sum of the exponents of $W_{f} W_{g}^{-1}$.

\section{Computations: subgroups of $S L_{2}(\mathbb{Z})$}

The following lemma is known. It can be proved directly, using standard combinatorial group theory algorithms (as implemented, for example, on the program GAP). In fact, $\phi\left(H_{3}\right)$ can be identified with the pre-image of the upper-triangular matrices in $\mathrm{PSL}_{2}(\mathbb{Z} / 3)$ under the natural reduction map, and $\phi\left(H_{4}\right)$ is conjugate in $\mathrm{SL}_{2}(\mathbb{R})$ to the kernel of the map to $\mathrm{PSL}_{2}(\mathbb{Z} / 2)$. It follows that $H_{3}$ has index 8 , and $H_{4}$ has index 12. For variety, we shall give a proof based on the Euclidean algorithm.

Lemma 6.1. The subgroups $H_{3}$ and $H_{4}$ have finite index in $\mathcal{M}_{1}^{1}$.

Proof. We shall prove the statement for $H_{4}$, the proof for $H_{3}$ being entirely analogous.

Given an ordered pair of relatively prime, non-zero integers $(m, n)$, we may generate a sequence $\left(m_{0}, n_{0}\right), \ldots,\left(m_{k}, n_{k}\right)$ recursively, as follows:

Let $\left(m_{0}, n_{0}\right)=(m, n)$. Suppose $\left(m_{i}, n_{i}\right)$ has been defined. If $\left|n_{i} \pm m_{i}\right|<$ $\left|n_{i}\right|$, then let $\left(m_{i+1}, n_{i+1}\right)=\left(m_{i}, n_{i} \pm m_{i}\right) ;$ if $\left|n_{i}+m_{i}\right| \geq\left|n_{i}\right|$ and $\mid n_{i}-$ $m_{i}|\geq| n_{i} \mid$ and $\left|m_{i} \pm 4 n_{i}\right|<\left|m_{i}\right|$, then let $\left(m_{i+1}, n_{i+1}\right)=\left(m_{i} \pm 4 n_{i}, n_{i}\right)$; if neither of these conditions holds, then terminate the sequence at $\left(m_{i}, n_{i}\right)$.

Claim. For any pair of relatively prime, non-zero integers $(m, n)$, the above rule defines a finite sequence terminating in $( \pm 1,0)$ or $(0, \pm 1)$.

Proof of claim. Suppose that $\left(m_{i}, n_{i}\right)$ has been defined, that neither $m_{i}$ nor $n_{i}$ is zero, and that $\left|n_{i}+m_{i}\right|$ and $\left|n_{i}-m_{i}\right|$ are both as big as $\left|n_{i}\right|$. Then $\left|m_{i}\right| \geq 2\left|n_{i}\right|$. Since $m$ and $n$ are relatively prime, it follows that $m_{i}$ and $n_{i}$ are relatively prime, and thus we have strict inequality $\left|m_{i}\right|>2\left|n_{i}\right|$, and so $\left|m_{i} \pm 4 n_{i}\right|<\left|m_{i}\right|$. So the sequence continues until we reach $(j, 0)$ or $(0, j)$. In this case, both $m$ and $n$ are divisible by $j$, so we have $j= \pm 1$. This proves the claim.

Now, $\mathrm{SL}_{2}(\mathbb{Z})$ acts on the hyperbolic plane, and there is an induced action on the circle at infinity, which is identified with $\mathbb{R} \cup\{\infty\}$.

Identifying the rational number $m / n$ with the vector $\left(\begin{array}{c}m \\ n\end{array}\right)$, and $\infty$ with $\left(\begin{array}{l}1 \\ 0\end{array}\right)$, the action of $\left\langle D_{x *}, D_{y *}^{4}\right\rangle$ on $\mathbb{Q} \cup \infty$ is given by

$$
\left(\begin{array}{cc}
1 & \pm 1 \\
0 & 1
\end{array}\right)\left(\begin{array}{c}
m \\
n
\end{array}\right)=\left(\begin{array}{c}
m \pm n \\
n
\end{array}\right) \quad \text { and } \quad\left(\begin{array}{cc}
1 & 0 \\
\pm 4 & 1
\end{array}\right)\left(\begin{array}{c}
m \\
n
\end{array}\right)=\left(\begin{array}{c}
m \\
\pm 4 m+n
\end{array}\right) .
$$


Therefore, by the claim, the orbit $\left\langle D_{x *}, D_{y *}^{4}\right\rangle\{0, \infty\}$ is dense in $\mathbb{R} \cup\{\infty\}$. Thus the domain of discontinuity of $\left\langle D_{x *}, D_{y *}^{4}\right\rangle$ is empty, and so $\left\langle D_{x *}, D_{y *}^{4}\right\rangle$ is a finite-index subgroup of $\mathrm{SL}_{2}(\mathbb{Z})$. Thus $H_{4}$ has finite index in $\mathcal{M}_{1}^{1}$.

Computation of exponent sums. Given an element $g \in \mathcal{M}_{1}^{1}$, let $m_{i}$ be the smallest positive integer such that $g^{m_{i}} \in H_{i}$. Thus there is word $W$ (not necessarily unique) such that $g_{*}^{m_{i}}=W\left(D_{x *}, D_{y *}^{i}\right)$. Let $n_{i}$ be the exponent sum of $D_{x *}$ in $W$. The numbers $m_{i}$ and $n_{i}$ can be computed on GAP, by using Reidemeister-Schreier style algorithms for subgroup presentations.

The computation of $m_{i}$ is quite straightforward. The computation of $W_{i}$ (and hence $n_{i}$ ) requires a slightly more complicated, but standard, procedure. The idea is to have GAP compute a presentation for the subgroup of $\mathrm{SL}_{2}(\mathbb{Z})$ generated by $D_{x *}, D_{y *}^{i}$ and $g_{*}^{n_{i}}$. After simplifying, GAP finds that the generator $g_{*}^{n_{i}}$ is redundant and returns the word $W$. Details can be found in the source code at www.math.buffalo.edu/ jdmaster.

\section{Computations: slopes}

Given an arbitrary $f \in \mathcal{M}_{1}^{1}$, we may associate slopes $\beta_{3}^{1}, \beta_{3}^{2}$ and $\beta_{4}^{1}, \beta_{4}^{2}$ for the boundary of a cyclic cover $M_{f^{m}}$, as follows.

To compute the slopes $\beta_{i}^{1}$, We first compute an integer $m$ such that $f^{m} \in H_{3} \cap H_{4}$. We then compute words $W_{3}$ and $W_{4}$ such that $f_{*}^{m}=W_{i}\left(D_{x *}, D_{y *}^{i}\right)$, as described in the previous section, and let $n_{i}$ be the exponent sum of $D_{x *}$ in $W_{i}$. We then let $g_{i}=W_{i}\left(D_{x}, D_{y}^{i}\right) \in \mathcal{M}_{1}^{1}$. Since $g_{i} \in J_{i}$, then by the proof of Theorem 1.2, we see that associated to the bundle $M_{g_{i}}$ are slopes $\beta_{3}^{1 \prime}=\left(3, n_{3}\right) / \operatorname{gcd}\left(3, n_{3}\right), \beta_{4}^{1 \prime}=\left(2, n_{4}\right) / \operatorname{gcd}\left(2, n_{4}\right)$ and $\beta_{4}^{2 \prime}=(1,0)$.

We use Lemma 5.2 to compute $t\left(f^{m} g_{i}^{-1}\right)$, and then use Lemma 5.1 to compute that

$$
\begin{aligned}
& \beta_{3}^{1}=\frac{\left(3, n_{3}-3\left(t\left(f^{m} g_{3}^{-1}\right)\right)\right)}{\operatorname{gcd}\left(3, n_{3}\right)} \\
& \beta_{4}^{1}=\frac{\left(2, n_{4}-2\left(t\left(f^{m} g_{4}^{-1}\right)\right)\right)}{\operatorname{gcd}\left(2, n_{4}\right)} \\
& \beta_{4}^{2}=\left(1,-t\left(f^{m} g_{4}^{-1}\right)\right)
\end{aligned}
$$

To compute $\beta_{3}^{2}$, we first use Lemmas 4.3 and 4.5 to compute the Alexander polynomial for the relevant cover of the manifold $M_{g_{3}}$. Then, 
as in the proofs of Corollaries 4.4 and 4.6, we obtain the slope $\beta_{3}^{2 \prime}$. Finally, using Lemma 5.1, we compute the slope $\beta_{3}^{2}$ for the manifold $M_{f^{m}}$.

If $\left\{\beta_{3}^{1}, \beta_{3}^{2}\right\} \cap\left\{\beta_{4}^{1}, \beta_{4}^{2}\right\}=\emptyset$, then we report "success," meaning that $M_{f}$ has a finite cover satisfying property $\mathrm{VH}$.

\section{Example}

Let $f=D_{x}^{-1} D_{y}$, so $M_{f}$ is the figure-eight knot exterior. We shall show that $f^{12}$ has property VH. Using GAP, we compute: $m=12$, so $f^{12} \in H_{3} \cap H_{4}$; also $W_{3}(a, b)=\left(a^{-1} b a b a^{-1} b a b\right)^{3}$ and $W_{4}(a, b)=\left(a^{-2} b^{-1} a^{-1} b^{-1} a^{-1}\right)^{4}$, and so $n_{3}=0$ and $n_{4}=-16$. We have $f_{*}^{12}=W_{3}\left(D_{x *}, D_{y *}^{3}\right)=W_{4}\left(D_{x *}, D_{y *}^{4}\right)$. Letting $g_{i}=W_{i}\left(D_{x}, D_{y}^{i}\right)$, then the slopes associated to $M_{g_{i}}$ are $\beta_{3}^{1 \prime}=(1,0)$, $\beta_{4}^{1 \prime}=(1,-8)$ and $\beta_{4}^{2 \prime}=(1,0)$.

The Alexander polynomial, $\Delta_{3}(s)$, for the relevant cover of $M_{g_{3}}$ is given by:

$$
\Delta_{3}(s)=q s^{4}+3 q s^{3}+2 q s^{2}+2 q s-q+q s^{-1}-2 q s^{-2}-2 q s^{-3}-3 q s^{-4}-q s^{-5} .
$$

This polynomial is non-monic whenever $|q|>1$. Thus, associated to $M_{g_{3}}$ is the slope $\beta_{3}^{2 \prime}=(1,0)$. We use Lemma 5.2 to compute that $t\left(f^{12} g_{3}^{-1}\right)=-3$ and $t\left(f^{12} g_{4}^{-1}\right)=4$.

Then

$$
\begin{aligned}
\beta_{3}^{1} & =\frac{\left(3, n_{3}-3\left(t\left(f^{m} g_{3}^{-1}\right)\right)\right)}{\operatorname{gcd}\left(3, n_{3}\right)}, \\
& =(1,3) \\
\beta_{3}^{2} & =\left(1,-t\left(f^{m} g_{3}^{-1}\right)\right) \\
& =(1,3) \\
\beta_{4}^{1} & =\frac{\left(2, n_{4}-2\left(t\left(f^{m} g_{4}^{-1}\right)\right)\right)}{\operatorname{gcd}\left(2, n_{4}\right)}, \\
& =(1,-12) \\
\beta_{4}^{2} & =\left(1,-t\left(f^{m} g_{4}^{-1}\right)\right) \\
& =(1,-4)
\end{aligned}
$$

Since $\left\{\beta_{3}^{1}, \beta_{3}^{2}\right\} \cap\left\{\beta_{4}^{1}, \beta_{4}^{2}\right\}=\emptyset$, then $M_{f^{12}}$ has property $\mathrm{VH}$. 


\section{An infinite family with property VH}

Proof of Theorem 1.4. Let $f_{n}=\left(D_{x}^{-1} D_{y}\right)^{12} D_{y}^{12 n}$. It may easily be checked that the induced map on $H_{1}(F)$ has trace bigger than 3 , and so the corresponding bundle $M_{f_{n}}$ is atoroidal. For $f_{0}$, a computation (see previous section) gives $\beta_{3}^{1}=(1,3), \beta_{3}^{2}=(1,3), \beta_{4}^{1}=(1,-12)$ and $\beta_{4}^{2}=(1,-4)$.

The element $f_{n}$ is equivalent in $\mathrm{SL}_{2}(\mathbb{Z})$ to the element

$$
g_{n}=\left(D_{x}^{-1} D_{y}^{3} D_{x} D_{y}^{3} D_{x}^{-1} D_{y}^{3} D_{x} D_{y}^{3}\right)^{3} D_{y}^{12 n} .
$$

One checks that the Alexander polynomial for the relevant cover of $M_{g_{n}}$ is equivalent $\bmod n$ to the Alexander polynomial for the relevant cover of the bundle with monodromy $D_{x}^{-1} D_{y}^{3} D_{x} D_{y}^{3} D_{x}^{-1} D_{y}^{3} D_{x} D_{y}^{3}$. One computes that the leading coefficient of the latter polynomial is $q$, and thus the leading coefficient of the former polynomial is divisible by $q$. Thus for the manifold $M_{g_{n}}$ we have slopes $\beta_{3}^{1 \prime}=\beta_{3}^{2 \prime}=(1,0)$. One computes $t\left(g_{n} f_{n}^{-1}\right)=-3$, and so for $f_{n}$, we have $\beta_{3}^{1}=\beta_{3}^{2}=(1,3)$.

Also, $f_{n}$ is equivalent in $\mathrm{SL}_{2}(\mathbb{Z})$ to the element $h_{n}=\left(D_{x}^{-2} D_{y}^{-4} D_{x}^{-1} D_{y}^{-4}\right.$ $\left.D_{x}^{-1}\right)^{4} D_{y}^{12 n}$. For the manifold $M_{h_{n}}$ we have $\beta_{4}^{2 \prime}=(1,0)$, and we compute $\beta_{4}^{1 \prime}=(1,-8)$. We compute $t\left(h_{n} f_{n}^{-1}\right)=4$, and so for $f_{n}$, we have $\beta_{4}^{1}=$ $(1,-12)$, and $\beta_{4}^{2}=(1,-4)$.

Since $\left\{\beta_{3}^{1}, \beta_{3}^{2}\right\} \cap\left\{\beta_{4}^{1}, \beta_{4}^{2}\right\}=\emptyset$, then for all $n>0$, the manifold $M_{f_{n}}$ is finitely covered by a bundle with property VH, by Theorem 1.2. Furthermore, the manifolds $M_{f_{n}}$ are all obtained by doing surgery on the same hyperbolic knot $K \subset M_{f_{0}}$. Therefore, by the results of [7, Section 3], it follows that there are infinitely many non-commensurable manifolds in the family $\left\{M_{f_{n}}\right\}$.

\section{Computer results}

For every monodromy of complexity at most 5 , the computer verified that a "success" criterion was met, and so the associated bundle is commensurable to one with property VH. The data for monodromies of complexity at most 5 is given below. Since we are only considering bundles up to commensurability, we have left out monodromies which are proper powers and 


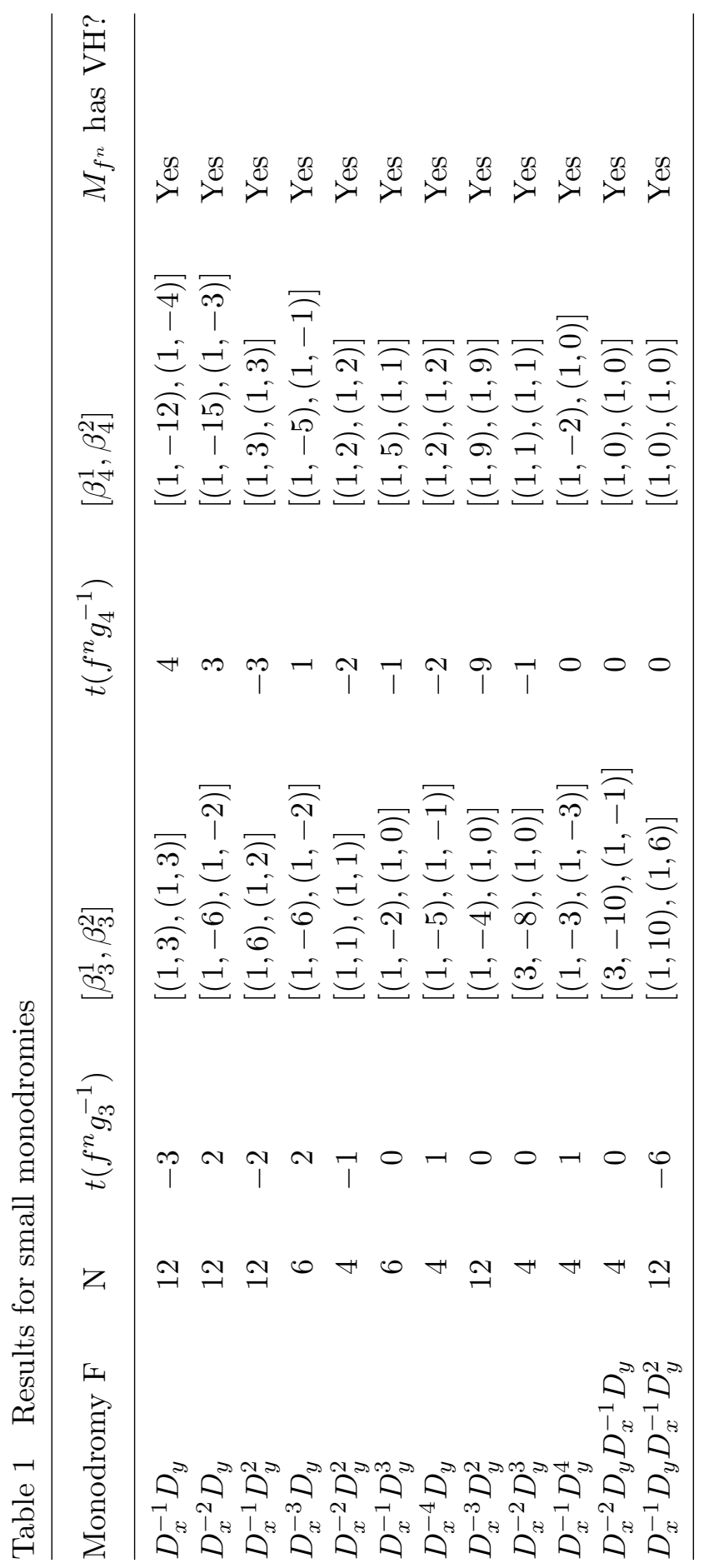


monodromies with negative trace. Also, we have only included words up to cyclic permutations. The " $n$ " in column two is the smallest positive integer such that $f^{n} \in H_{3} \cap H_{4}$.

From table 1 we see that every bundle of complexity at most five is commensurable to a bundle with property $\mathrm{VH}$.

We considered all cyclically reduced, primitive, positive words on $D_{x}^{-1}$ and $D_{y}$ of length at most 12 , representing hyperbolic monodromies. There are 745 of these. We verified that all but 36 of the associated bundles are finitely covered by a bundle with property VH. Here we have not distinguished conjugate classes in $\mathrm{SL}_{2}(\mathbb{Z})$, so these words do not all correspond to distinct bundles. The monodromy of smallest complexity which we cannot handle is $D_{x}^{-3} D_{y}^{3}$, for which $\beta_{3}^{1}=\beta_{4}^{1}=(1,-3)$.

The routine runs quickly on words of rather large size. For example, for the monodromy $f=D_{x}^{11} D_{y}^{3} D_{x} D_{y}^{6} D_{x}^{-4}\left(D_{y} D_{x}^{-1}\right)^{4} D_{y}$, a few seconds' computation gives $f^{4} \in H_{3} \cap H_{4}$, with associated slopes $\beta_{3}^{1}=(1,-17), \beta_{3}^{2}=(1,-15)$ and $\beta_{4}^{1}=(1,-18), \beta_{4}^{2}=(1,-18)$. Thus $M_{f^{4}}$ has property $\mathrm{VH}$.

\section{Acknowledgment}

Thanks are due to Genevieve Walsh for pointing out an error in a previous version, and to the referees for their detailed and helpful suggestions.

\section{References}

[1] M. Baker, Covers of Dehn fillings of once-punctured torus bundles II, Proc. Am. Math. Soc. 110 (4), (1990), 1099-1108.

[2] S. Boyer and X. Zhang, Virtually Haken 3-manifolds and Dehn filling, Topology 39(1), (2000), 103-114.

[3] D. Cooper and D.D. Long, Virtually Haken Dehn filling, J. Differential Geom. 52 (1999), 173-187.

[4] D. Cooper and G. Walsh, Virtual Haken fillings and semi-bundles, Pre-print, 2004, arxiv:math.GT/0407328.

[5] N. Dunfield and W.P. Thurston, The virtual Haken conjecture: experiments and examples, Geom. Topol. 7 (2003), 399-441.

[6] D.D. Long and A.W. Reid, Integral points on character varieties, Math. Ann. 325(2), (2003), 299-321. 
[7] J.D. Masters, Virtual homology of surgered torus bundles, Pacific J. Math. 197(1), (2001), 205-223.

[8] E.H. Spanier, Algebraic topology, Springer-Verlag, New York, 1966.

Mathematics Department

SUNY AT BUFFALO

Buffalo, NY 14260-2900

USA

E-mail address: jdmaster@buffalo.edu

Received March 20, 2006 